\title{
Effects of the Tensor Force on the Multipole Response in Finite Nuclei
}

\author{
Li-Gang Cao ${ }^{1,2,3,4,5}$, G. Colò ${ }^{1,2,5}$, H. Sagawa ${ }^{6}$, P.F. Bortignon ${ }^{1,2}$, and L. Sciacchitano ${ }^{1}$ \\ ${ }^{1}$ Dipartimento di Fisica, Università degli Studi di Milano, via Celoria 16, Milano, Italy \\ ${ }^{2}$ Istituto Nazionale di Fisica Nucleare (INFN), Sez. di Milano, via Celoria 16, Milano, Italy \\ ${ }^{3}$ Institute of Modern Physics, Chinese Academy of Science, Lanzhou 730000, P.R. China \\ ${ }^{4}$ Center of Theoretical Nuclear Physics, National Laboratory of Heavy Ion Accelerator of Lanzhou, Lanzhou 730000, P.R. China \\ ${ }^{5}$ Kavli Institute for Theoretical Physics China, CAS, Beijing 100190, P.R. China and \\ ${ }^{6}$ Center for Mathematics and Physics, University of Aizu, Aizu-Wakamatsu, Fukushima 965-8560, Japan
}

\begin{abstract}
We present a thorough analysis of the effects of the tensor interaction on the multipole response of magic nuclei, using the fully self-consistent Random Phase Approximation (RPA) model with Skyrme interactions. We disentangle the modifications to the static mean field induced by the tensor terms, and the specific features of the residual particle-hole $(\mathrm{p}-\mathrm{h})$ tensor interaction, for quadrupole $\left(2^{+}\right)$, octupole $\left(3^{-}\right)$, and also magnetic dipole $\left(1^{+}\right)$responses. It is pointed out that the tensor force has a larger effect on the magnetic dipole states than on the natural parity states $2^{+}$ and $3^{-}$, especially at the mean field level. Perspectives for a better assessment of the tensor force parameters are eventually discussed.
\end{abstract}

PACS numbers: 21.60.Jz, 21.65.Ef, 24.30.Cz, 24.30.Gd

\section{INTRODUCTION}

The nuclear effective interactions like the zero-range Skyrme forces have been quite successful in many respects since they have been introduced, mainly because of continuous efforts to apply them to the study of different nuclear phenomena, and to improve their form and their parameters. The Skyrme forces contain typically 10 free parameters which are fitted to reproduce empirical bulk properties of uniform nuclear matter, and masses and radii of a few magic nuclei. These forces, or at least several of the many existing parameter sets, describe in a reasonable way the global features of the ground-state properties along the nuclear chart (i.e., binding energies, radii, deformations). In some cases one needs to input more information in the parameter fitting: the Lyon forces [1] have been for instance determined by requiring a reproduction of the equation of state of neutron matter emerging from realistic calculations. Properties of excited states (vibrations, rotations) have been studied using the Skyrme parameter sets, allowing a large amount of physical insight. For a review on mean-field calculations the reader can consult Ref. [2]. Few ideas about selecting the Skyrme forces which have reasonable overall performances can be found in [3]. Last but not least, in the quest for a universal local Energy Density Functional (EDF) for nuclei, the Skyrme framework is often used as a starting point.

Of course, there are drawbacks and limitations of the mean-field approaches based on effective forces. This issue should be discussed together with the answer to the question whether we need to generalize the existing Skyrme parametrizations. In this spirit, since most of the Skyrme sets which have been widely used are purely central, many groups have recently devoted much attention to the role played by the zero-range tensor terms that can be added to the standard Skyrme terms (see Refs. [4, 5, 6, 7, 8, 9, 10, 11]). This blooming of theoretical studies has followed the claim by the authors of Ref. [12], that the tensor force is crucial for the understanding of the evolution of the single-particle energies in exotic nuclei.

Certainly, the mentioned studies have elucidated that the tensor force does play a role. At the same time, the single-particle states are not the right observable on which to constrain an effective force or an effective functional, since for these states other correlations beyond mean-field are expected to manifest themselves.

It is certainly quite timely to analyze in detail how the tensor terms added to a standard Skyrme force, or the new parameter sets introduced in [8] (fitted with the tensor included), behave as far as the excited states are concerned. The study of nuclear collective vibrations, within self-consistent Random Phase Approximation (RPA) on top of Hartree-Fock (HF), is a well-defined framework which has allowed testing the effective Skyrme sets for many years. Recently, we have developed fully self-consistent RPA [13], and quasi-particle RPA (QRPA) [14], schemes. The full self-consistency, in the nonrelativistic framework, is essential to respect theorems concerning sum rules associated with appropriate operators. In this paper, we extend these schemes by including the tensor terms both in HF and in RPA as terms of the residual interaction, and we analyze the results in the case of the multipole response of magic nuclei.

Our work is the first one which attempts a detailed explanation of the effects induced by the tensor force on the response of finite nuclei within the Skyrme framework. However, the fact that the subject is of interest at present is testified by recent papers that are devoted to related topics. V. De Donno et al. [15] have studied the lowlying magnetic excitations in several nuclei, either within a phenomenological approach or by using the Gogny force. 
The authors of [16] have investigated self-consistently, using the Skyrme forces and the zero-range tensor terms, the response of uniform matter.

The outline of our paper is the following. Since Skyrme-RPA is a well-known framework we will survey very briefly our formalism in Sec. [I] More emphasis is given to the discussion of the results, for quadrupole, octupole and magnetic dipole states, in Sec. III. An overall conclusion, together with some perspectives for future work on the tensor force and on possible constraints on its parameters, is given in Sec. IV The main technical part of this work, namely the evaluation of the $\mathrm{p}$ - $\mathrm{h}$ matrix elements of the tensor interaction, is described in Appendix $\mathrm{A}$, while Appendix B] describes and approximate yet quite useful separable approximation for the tensor force that is used as a guideline to understand some features of our numerical results.

\section{FORMALISM}

As mentioned in the Introduction, Skyrme-RPA theory is well known since many years, especially in its matrix formulation. We have been using for several applications a scheme which is fully self-consistent, that is, where there is no approximation in the residual interaction since all its terms are taken into account including the two-body spinorbit and Coulomb. In our scheme we use box boundary conditions. In other words, the continuum is discretized. In the present case the box radius is set at $15 \mathrm{fm}$ for the Ca isotopes and at $20 \mathrm{fm}$ for ${ }^{208} \mathrm{~Pb}$.

After solving the HF equations in coordinate space and determining, together with the mean field, the unoccupied states as eigenstates of this mean field in the mentioned large box, we build up a model space of p-h configurations with given multipolarity $J^{\pi}$ and we write and solve the RPA matrix equation in that space. The model space includes the configurations built up with all hole states, and with the particle states labelled by $n_{p}, l_{p}$ and $j_{p}$ (number of radial nodes, spatial and total angular momentum); $l_{p}$ and $j_{p}$ take all possible values which are allowed by selection rules, while $n_{p}$ varies between $n_{\max }+1\left(n_{\max }\right.$ is the number of nodes of the last occupied state) and $n_{\max }+1+\Delta n$.

The value of $\Delta n$ is chosen large enough, so that the isoscalar (IS) EWSR for the multipoles $2^{+}, 3^{-}$exhaust practically all $(\sim 97-99 \%)$ the double commutator (DC) value. In particular, we use $\Delta n=10$ unoccupied shells in the case of the $2^{+}$and $1^{+}$calculations in ${ }^{40} \mathrm{Ca}$ and ${ }^{48} \mathrm{Ca}, \Delta n=9$ unoccupied shells in the case of the $3^{-}$calculations in the same nuclei, and $\Delta n=8$ unoccupied shells for ${ }^{208} \mathrm{~Pb}$.

The eigenvalues $E_{n}$ and eigenvectors $|n\rangle$ of the RPA equations allow calculating the response function to different operators. In particular we are going in what follows to show figures with the strength function associated with the operator $\hat{F}$, namely

$$
S(E)=\sum_{n}|\langle n|\hat{F}| 0\rangle|^{2} \delta\left(E-E_{n}\right),
$$

where the sum spans the whole RPA spectrum. In the case of discretized RPA one has in reality sharp peaks but in the figures we shall display $S(E)$ smoothed out using Lorentzian functions (having $1 \mathrm{MeV}$ width). We shall use isoscalar quadrupole and octupole operators, that is,

$$
\hat{F}_{L}=\sum_{i=1}^{A} \sqrt{2 L+1} r_{i}^{L} Y_{L M}\left(\hat{r}_{i}\right)
$$

with $L=2$ and 3 , respectively. We focus our study on the $1^{+}$spin-flip states as well. In this case the following operators are used, namely

$$
\hat{F}_{J}(I S)=\sum_{i=1}^{A} \frac{g^{I S} e \hbar}{2 m c} \sqrt{J(2 J+1)} \sigma_{i}^{\mu} Y_{00}\left(\widehat{r}_{i}\right)
$$

in the isoscalar case, and

$$
\hat{F}_{J}(I V)=\sum_{i=1}^{A} \frac{g^{I V} e \hbar}{2 m c} \sqrt{J(2 J+1)} \sigma_{i}^{\mu} Y_{00}\left(\widehat{r}_{i}\right) \tau_{i}^{z}
$$

in the isovector case. We shall consider the case $J=1$. In the above equations the nuclear magneton $\mu_{N}=\frac{e \hbar}{2 m c}$ appears together with the quantities $g^{I S}=\frac{1}{4}\left(g_{s}^{n}+g_{s}^{p}-1\right)=0.19$, and $g^{I V}=\frac{1}{4}\left(g_{s}^{n}-g_{s}^{p}+1\right)=-2.10$.

We note that the tensor contribution must not alter the DC value for the EWSRs in the case of the operators (2), for all values of $L$. It does change, instead, the value of the EWSRs in the case of the operators (3) and (4) (cf., e.g., Ref. [17]). 
Two sets of Skyrme parameters are employed in the calculations presented below. On the one hand, we have chosen the set SLy5 [1] and supplemented it with tensor terms characterized by the values chosen in [6] and used in [10] as well. The analysis of these results will elucidate qualitatively the role played by the tensor terms, but in order to compare with the case of a force having all parameters fitted on equal footing (including those of the tensor terms) we have performed also calculation with the set T44 introduced in Ref. [8]. For the reader's convenience, all the

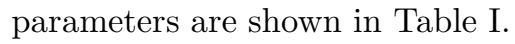

\section{RESULTS}

\section{A. Quadrupole response}

Fig. 1 displays in the upper panel the IS response of the nucleus ${ }^{40} \mathrm{Ca}$. For a $\vec{l} \cdot \vec{s}$-closed nucleus (that is, a nucleus where both spin-orbit partner states are occupied), and a natural-parity excitation spectrum, we do not expect that the tensor interaction plays any significant role. In particular, for the ground state, the changes in the spin-orbit splittings due to the tensor terms have been written in previous works and they depend on the so-called spin-orbit densities defined as

$$
J_{q}(r)=\frac{1}{4 \pi r^{3}} \sum_{i}\left(2 j_{i}+1\right)\left[j_{i}\left(j_{i}+1\right)-l_{i}\left(l_{i}+1\right)-\frac{3}{4}\right] R_{i}^{2}(r)
$$

where the isospin quantum number $q=0(1)$ labels neutrons (protons) and the index $i=n, l, j$ runs over all states having the given $q . R_{i}(r) \equiv \frac{u_{i}(r)}{r}$ is the radial part of the wavefunction. So, our expectation is obviously based, in the unperturbed case, on the fact that $J_{q}$ is negligibly small (both for neutrons and for protons) in the case at hand. In fact, the microscopic calculations show that the results are exactly the same, with and without the tensor force.

For comparison, we have computed the same IS response in the nucleus ${ }^{48} \mathrm{Ca}$ and the results are shown in the middle panel of Fig. 1. The response in the giant resonance region is not affected strongly by the inclusion of the tensor force. Although some changes are found in the high-energy part of the strength function, the main impact of the tensor terms is visible in the states below the isoscalar giant quadrupole resonance (ISGQR). For the sake of clarity, the properties of the lowest $2^{+}$states are shown in Table II We have analyzed the changes of these properties induced by the tensor force. With the interaction SLy5, and the tensor parameters $T$ and $U$ already used in Refs. [6, 10], the spin-orbit splittings are increased. Normally, at least for well-bound states, the splittings should increase more for larger values of $l$. Consequently, the low-lying $2^{+}$state in ${ }^{48} \mathrm{Ca}$, which is mainly due to the neutron $\mathrm{f}_{7 / 2} \rightarrow$ $\mathrm{p}_{3 / 2}$ transition, is pushed upward in the unperturbed response by the tensor force. We denote this shift by $\Delta E_{\mathrm{HF}}$. The effect of the residual tensor force $V_{\text {tensor }}$ included in RPA can be estimated by means of

$$
\Delta E_{\mathrm{RPA}} \approx \Delta E_{\mathrm{HF}}+\left\langle V_{\text {tensor }}\right\rangle
$$

where $\Delta E_{\mathrm{RPA}}$ indicates the difference between the RPA result with and without the tensor force, and \langle\rangle means that we extract here an average value of the residual force. In our SLy5 calculation, the value of $\Delta E_{\mathrm{HF}}$ is $1.41 \mathrm{MeV}$ and from the shift of the RPA peak $(0.83 \mathrm{MeV})$ we extract $\left\langle V_{\text {tensor }}\right\rangle=-0.58 \mathrm{MeV}$.

The fact that the residual force is attractive, albeit not large, can be understood using the argument developed in Appendix B. Using a separable approximation for the tensor interaction, it is shown that both the tensor-even and tensor-odd terms produce indeed attraction in the present case, where $T$ is positive and $U$ is negative [cf. Eqs. (B9) and $[\overline{B 13}]$.

Our understanding needs to be confirmed by another example. In the lower panel of Fig. 1, the IS response for the nucleus ${ }^{208} \mathrm{~Pb}$ is displayed. There is essentially no effect of the tensor force in the giant resonance region. The low-lying $2^{+}$state is moved upward in the calculation with tensor by $\Delta E_{\mathrm{RPA}}=0.17 \mathrm{MeV}$. However, if we look at the centroid of the low-energy unperturbed response, the shift due to the tensor force is $\Delta E_{\mathrm{HF}}=0.60 \mathrm{MeV}$. Then, from Eq. (6) we extract a value of $\left\langle V_{\text {tensor }}\right\rangle=-0.43 \mathrm{MeV}$, that is, consistent with what extracted in the case of ${ }^{48} \mathrm{Ca}$ and with the qualitative argument of Appendix B

The inclusion of the tensor force on top of SLy5, with the parameters used in our previous works [6, 10], does not improve the agreement with experimental values. It is desirable to check the behavior of a different interaction, and we have chosen one of those which have been fitted including the tensor terms in Ref. [8], that is, the set named T44.

In this case, not so much emphasis should be put on the discussion of the effect of the tensor terms separately from that of other Skyrme parameters, since all parameters have been fitted together. We just briefly point out that the tensor residual interaction is almost negligible in this case. In fact, for the low-lying $2^{+}$state of ${ }^{48} \mathrm{Ca}$ calculated with T44, the values of the quantities defined in Eq. (6) are $\Delta E_{\mathrm{HF}}=-0.13 \mathrm{MeV}, \Delta E_{\mathrm{RPA}}=-0.18 \mathrm{MeV}$ and $\left\langle V_{\text {tensor }}\right\rangle=-0.05$ 
$\mathrm{MeV}$. The same values are $\Delta E_{\mathrm{HF}}=-0.54 \mathrm{MeV}, \Delta E_{\mathrm{RPA}}=-0.55 \mathrm{MeV}$ and $\left\langle V_{\text {tensor }}\right\rangle=-0.01 \mathrm{MeV}$ in the case of ${ }^{208} \mathrm{~Pb}$. We should also mention that in the case of infinite matter [16] the effect of the tensor terms has been found to be small, in the case of the Tij parameter sets of Ref. [8], looking at the $S=0$ response both with and without the tensor.

In conclusion, the behavior of the tensor interaction in the quadrupole response can be fairly well understood. Its effects are visible only for the low-lying states (not for giant resonances) and are mainly related to the mean field since the residual tensor interaction matrix elements are either tiny or anyway not very large. We end this Section by adding that the isovector response has also been computed, although it is not displayed in our figures. We have in fact observed that the centroid energy of the (high-lying) isovector strength may change by few hundreds of keV and only in few cases by about $\approx 1 \mathrm{MeV}$ when the tensor force is included.

\section{B. Octupole response}

In a similar style as for the quadrupole case, the IS octupole responses of the nuclei ${ }^{40} \mathrm{Ca},{ }^{48} \mathrm{Ca}$ and ${ }^{208} \mathrm{~Pb}$ are displayed in the upper, middle and lower panels of Fig. 2, respectively. Also in the present case, we do not discuss the IV strength (for which a comment similar to the one made at the end of the previous subsection applies) but only the IS strength.

The detailed behavior of the low-lying states is shown in Table III In the case of ${ }^{40} \mathrm{Ca}$, as we have already commented in the previous subsection, there is no effect of the tensor terms at the mean field level since $J_{p}$ and $J_{n}$ are negligibly small. In the case of SLy5 plus the $T$ and $U$ values of [6, 10], the value of $\left\langle V_{\text {tensor }}\right\rangle$ can be therefore deduced from the shift of the RPA energy of the lowest $3_{1}^{-}$state, and it is $-0.76 \mathrm{MeV}$, not much different from the values obtained in the case of the low-lying $2^{+}$(cf. the previous subsection).

In the case of ${ }^{48} \mathrm{Ca}$, there is no systematic behavior in the shifts of the unperturbed p-h transitions when the tensor terms are included. In this nucleus $J_{p}$ is negligibly small while $J_{n}$ is positive due to the neutron $\mathrm{f}_{7 / 2}$ contribution. With the force SLy5, and the parameters $T$ and $U$ of [6, 10], the proton spin-orbit splittings are reduced, whereas the neutron ones are enlarged. The $3_{1}^{-}$state, which appears without tensor at $4.78 \mathrm{MeV}$ as it is shown in Table III, has the proton $\mathrm{s}_{1 / 2} \rightarrow \mathrm{f}_{7 / 2}$ and $\mathrm{d}_{3 / 2} \rightarrow \mathrm{f}_{7 / 2}$ as main components, and both are pushed upward in energy when the tensor is included. This explains the large positive shift of this RPA state, despite the (not strong) attractive contribution provided by the residual interaction. The same $\mathrm{p}$-h configurations are also the main ones which contribute to the second RPA state at $5.80 \mathrm{MeV}$ which is visible in Fig. 2 (in the curve corresponding to the calculation without tensor). However, in the case of other RPA states the situation is different. The third peak visible in Fig. 2 (once more, in the curve corresponding to the RPA calculation without tensor) lies at $9.23 \mathrm{MeV}$, and it receives contribution both from configurations which are pushed upward by the tensor inclusion, like the proton $\mathrm{d}_{3 / 2} \rightarrow \mathrm{p}_{3 / 2}$ and the neutron $f_{7 / 2} \rightarrow g_{9 / 2}$, and from others which are pushed downward like the neutron $d_{3 / 2} \rightarrow p_{3 / 2}$. So, the inclusion of the tensor cannot produce an effect which is understandable in simple terms. On top of this, we have verified that the RPA states calculated with the tensor included, contain somewhat different admixtures of p-h configurations with respect to those calculated without the tensor force.

Also in ${ }^{208} \mathrm{~Pb}$ several configurations contribute to the collective low-lying $3_{1}^{-}$state. This collectivity is enough, so that the effects coming from unperturbed configuration which are pushed upward or downward by the tensor terms, together with the effect of the tensor residual interaction, essentially cancel one another. Both from Fig. 2 and Table III one can see that the energies and $\mathrm{B}(\mathrm{E} 3)$ values in ${ }^{208} \mathrm{~Pb}$ are not affected significantly when tensor is included. The conclusion which can be reached, looking at the results obtained by using the SLy5 interaction and the tensor parameters from [6, 10], is that the effects of the tensor force on the octupole states are rather dependent on the specific nucleus, that is, on the relevant shell model states. In any case, we do not observe huge effects.

If we use the interaction T44, these qualitative conclusions remain valid, although the behavior of the tensor force is different in detail because of the contributions with different signs of $T$ and $U$, and their smaller absolute values. Actually, as in the quadrupole case, these differences do not manifest dramatically in the final results of the low-lying states in ${ }^{48} \mathrm{Ca}$ and ${ }^{208} \mathrm{~Pb}$. In the case of ${ }^{40} \mathrm{Ca}$, the gap in this nucleus turns out to be very small (about $3.3 \mathrm{MeV}$ ) as compared to the case of standard Skyrme forces which are fitted without tensor terms. In fact, this gap is, for example, $5.51 \mathrm{MeV}$ with SLy5. This explains the small energy of the low-lying $3^{-}$in ${ }^{40} \mathrm{Ca}$. In some cases this behavior of T44 might lead to RPA instabilities, which, however, we have not observed by studying these low multipole responses in finite nuclei.

\section{Magnetic dipole response}

On quite general grounds, it can be expected that the effects of the tensor force are larger for spin (or for spinisospin) states, both at the mean field level since unperturbed configurations are sensitive to the spin-orbit splittings, 
and also as far as RPA correlations are concerned. Some of the authors of the present work have already analyzed the role of the tensor correlations in the case of charge-exchange, spin-isospin states like the Gamow-Teller (GT) and spin-quadrupole resonances [20]. Tensor correlations are quite strong in that case, and lead to a lowering of the main GT peak by about $2 \mathrm{MeV}$ in ${ }^{90} \mathrm{Zr}$ and ${ }^{208} \mathrm{~Pb}$, which is accompanied by a strong repulsive shift of a sizeable fraction of strength (pushed at energies higher than the giant resonance region, at $30 \mathrm{MeV}$ and above). These effects are certainly more prominent than what has been discussed in the previous subsection for the non spin-flip transitions. Many earlier works in the literature have emphasized the role of the tensor force in the spin and spin-isospin channel, but not in a self-consistent framework like in the present case or in the case of Ref. [20]. The self-consistent calculation of the uniform matter response performed using the Skyrme force T44 in [16], confirms that larger and non-trivial effects from the tensor terms may be expected in the spin channel. With this background, we have made calculations for the magnetic dipole response in ${ }^{48} \mathrm{Ca}$ and ${ }^{208} \mathrm{~Pb}$.

The results we have obtained in the case of ${ }^{48} \mathrm{Ca}$ are displayed in Fig. 3. In the left panels, the strength functions associated with the SLy5 Skyrme set plus the $T$ and $U$ parameters of [6] are shown. The HF peak is associated with the neutron $\mathrm{f}_{7 / 2} \rightarrow \mathrm{f}_{5 / 2}$ configuration, which lies at $7.06 \mathrm{MeV}$ without the tensor contribution, and is pushed at 10.68 $\mathrm{MeV}$ by the inclusion of tensor terms. In the case of RPA, we deal with a somewhat undesired feature of the SLy5 set, which is characterized by a positive value of the Landau parameter $G_{0}=1.14$ and a negative value of $G_{0}^{\prime}=-0.15$. Repulsion in the spin-isoscalar (IS) channel and attraction in the spin-isovector (IV) channel is in contrast with the empirically accepted values of the Landau parameters, and, as already noticed in [21], constitutes an anomaly of the SLy5 set. In the case at hand, the residual interaction is essentially the $J^{\pi}=1^{+}$diagonal matrix element of the mentioned neutron configuration, that is, it is the sum of the IS and IV part. Without tensor, from the fact that the RPA peak is at $9.28 \mathrm{MeV}$ one can extract that the interaction is dominated by the IS contribution and is repulsive (its value being $2.22 \mathrm{MeV}$ ). Including the tensor interaction, the RPA peak moves to $12.31 \mathrm{MeV}$, so the shift due to tensor correlations is $3.03 \mathrm{MeV}$ : the tensor residual interaction can then be extracted from Eq. (6) and it turns out to be $-0.59 \mathrm{MeV}$. The fact that it is attractive is understandable in keeping with Eq. (B18) and from the negative sign of $U$.

In the case of the T44 interaction, the values of the Landau parameters $G_{0}$ and $G_{0}^{\prime}$ are, respectively, 0.40 and 0.06 . The results associated with this parameter set are visible in the right panels of Fig. 3, and one can see that the effect of the tensor is quite small as far as both unperturbed mean-field and RPA correlations are concerned. This is related to the smallness of the parameter $U$ [cf. Table I. Eq. (5) and the formulas of Appendix B]. In the case of the magnetic dipole state we have, therefore, found that different ways of including the tensor terms on top of the Skyrme interaction produce different effects. The experimental value of the M1 peak is $10.23 \mathrm{MeV}$ [22]. Since the theoretical values are 9.28 MeV (SLy5 without tensor), 12.31 MeV (SLy5 with tensor), 10.47 MeV (T44), it seems that the result obtained with T44 is preferable.

We turn to the analysis of the results for ${ }^{208} \mathrm{~Pb}$ which are reported in Figs. 4 and 5 , in the case of SLy5 plus the tensor force parameters of [6] and in the case of T44, respectively. In the unperturbed spectrum, the main role is played by the proton $h_{11 / 2} \rightarrow h_{9 / 2}$ and neutron $i_{13 / 2} \rightarrow i_{11 / 2}$ configurations. They are clearly visible in the upper panels of the two figures. In the case of the SLy5 interaction, without tensor, the proton configuration lies at 5.85 $\mathrm{MeV}$ while the neutron configuration is at $7.49 \mathrm{MeV}$. As already mentioned several times, in the case of SLy5 plus the the tensor parameters of [6] the spin-orbit splittings are increased when the tensor terms are taken into account: the energies become $6.45 \mathrm{MeV}$ and $9.17 \mathrm{MeV}$, respectively, for the proton and neutron configurations we have mentioned.

Within RPA, without tensor, we have found two peaks at $7.39 \mathrm{MeV}$ and $9.14 \mathrm{MeV}$. The lowest peak is mainly composed by the proton $\mathrm{h}_{11 / 2} \rightarrow \mathrm{h}_{9 / 2}$ configuration with an admixture of the neutron $\mathrm{i}_{13 / 2} \rightarrow \mathrm{i}_{11 / 2}$ configuration having different sign in its amplitude; the highest peak is mainly based on the neutron $i_{13 / 2} \rightarrow i_{11 / 2}$ configuration, with some admixture of the proton one, $h_{11 / 2} \rightarrow h_{9 / 2}$, having the same sign in its amplitude. In other words, the lowest (highest) state has more IV (IS) character. This isospin character is not strongly pronounced because, with the values of $T$ and $U$ that have been employed, the non-diagonal matrix element which mixes the proton and neutron configurations is small. Since experimentally one finds that the lowest (highest) state has more IS (IV) character, there is a doublet inversion which is related to the values of the Landau parameters (discussed above). We also notice that inversions of IS and IV spin doublet have also emerged from the self-consistent Gogny calculations of Ref. [15].

With the inclusion of the tensor terms, the lowest and highest peak move, respectively, to $7.79 \mathrm{MeV}$ and 10.57 $\mathrm{MeV}$. If we neglect the small mixing between the proton and neutron configurations, we can extract the values of the matrix elements of the residual tensor force from Eq. (6) separately for the proton and neutron states. We find, respectively, $-0.20 \mathrm{MeV}$ and $-0.25 \mathrm{MeV}$. These values are smaller but have the same sign as in ${ }^{48} \mathrm{Ca}$.

Similarly to ${ }^{48} \mathrm{Ca}$, the results in ${ }^{208} \mathrm{~Pb}$ obtained by using $\mathrm{T} 44$ seem better. In particular, we can notice that in this case one has the correct ordering in the spin doublet, the IS (IV) peak being the lowest (highest). In fact, with the values of $T$ and $U$ associated with this parameter set, the IS and IV characters of the states are more pronounced due to a larger value of the matrix element mixing neutrons and protons. The values of the energies are respectively 6.12 $\mathrm{MeV}$ and $8.27 \mathrm{MeV}$ and they compare better with the experimental findings (see e.g. [23] and references therein) 
which are $5.85 \mathrm{MeV}$ and $7.30 \mathrm{MeV}$, than SLy5 without tensor (which gives 7.39 MeV and 9.14 MeV) or SLy5 with tensor (which gives $7.79 \mathrm{MeV}$ and $10.57 \mathrm{MeV}$ ).

\section{CONCLUSION AND PERSPECTIVES}

The tensor component of the bare nuclear force has been always of central interest for nuclear physics, but as far as effective interactions for many-nucleon systems are concerned, only recently we have achieved some first understanding of its role within self-consistent frameworks. In particular, in recent years many works have appeared in the literature which deal with the effect of tensor terms when they are added on top to, or fitted together with, Skyrme-type forces. These works have dealt with the impact of tensor terms on bulk properties like masses, or on single-particle states.

Our work deals, for the first time, with the effects produced by these tensor terms on the multipole response of finite nuclei. We have attempted to disentangle the effects due to the modifications of the static mean field, and those due to the residual interaction, by analyzing the results of self-consistent HF plus RPA calculations performed in the case of quadrupole, octupole and magnetic dipole channels.

The modifications of the static mean field had been already understood by means of our previous works. The dominant character (attractive or repulsive) of the residual interaction matrix elements has been extracted from the numerical calculations but also understood on the basis of a separable approximation for the tensor p-h force. Then, it has become evident that since these two effects are governed by different combinations of the parameters of the tensor force, the effects which are visible on the final results are ruled by a delicate interplay and are non-trivial. This is one of the main findings of the present work.

The magnetic dipole states are more affected by the inclusion of the tensor terms at the mean field level, since unperturbed configurations correspond exactly to energy jumps between spin-orbit partners. The inclusion of the tensor residual interaction is demanded by self-consistency: for its matrix elements we have extracted typically values around few or several hundreds of $\mathrm{keV}$, but these values depend of course on the values of the tensor force parameters $T$ and $U$.

Our work is exploratory and we have shown that the tensor force plays a role in RPA since the matrix elements are in general not negligible. We have considered two cases, namely the parameter set SLy5 plus the tensor parameters of [ 6 ] and the set T44. The latter seems preferable if one looks at the comparison with experiment in the magnetic dipole case, but not for some of the low-lying states in Ca isotopes.

Actually, before learning more from the comparison with experiment, and fitting an ultimate Skyrme set including the tensor terms, one should ask the question whether the Skyrme ansatz is general enough for the spin-isospin channel. Some of the most modern, and most widely used, sets like the Lyon parameterizations have somewhat unsatisfactory values of the spin and spin-isospin Landau parameters and this anomaly should be cured. Improved Skyrme sets and Skyrme sets fitted with the tensor terms can be studied in detail, as far as their performance for excited states is concerned, by using the present RPA formalism. This is the main perspective opened by our present work.

\section{Acknowledgments}

C.L. acknowledges the support of the UniAMO fellowship provided by Fondazione Cariplo and Università degli Studi which has allowed his stay in Milano, and the support of the National Science Foundation of China under Grant Nos. 10875150. C.L. and G.C. also acknowledge partial support from the Kavli Institute for Theoretical Physics China (KITPC). This work is partially supported by the Japanese Ministry of Education, Culture, Sports, Science and Technology by Grant-in-Aid for Scientific Research under the program number (C(2)) 20540277.

\section{APPENDIX A: CALCULATION OF THE P-H MATRIX ELEMENTS OF THE TENSOR INTERACTION}

We use the triplet-even and triplet-odd zero-range tensor terms which have been introduced originally by Skyrme [24] and read

$$
\begin{aligned}
V_{\text {tensor }}= & \frac{T}{2}\left\{\left[\left(\sigma_{1} \cdot \mathbf{k}^{\prime}\right)\left(\sigma_{\mathbf{2}} \cdot \mathbf{k}^{\prime}\right)-\frac{\mathbf{1}}{\mathbf{3}}\left(\sigma_{\mathbf{1}} \cdot \sigma_{\mathbf{2}}\right) \mathbf{k}^{\prime} \mathbf{2}\right] \delta\left(\mathbf{r}_{\mathbf{1}}-\mathbf{r}_{\mathbf{2}}\right)+\delta\left(\mathbf{r}_{\mathbf{1}}-\mathbf{r}_{\mathbf{2}}\right)\left[\left(\sigma_{\mathbf{1}} \cdot \mathbf{k}\right)\left(\sigma_{\mathbf{2}} \cdot \mathbf{k}\right)-\frac{\mathbf{1}}{\mathbf{3}}\left(\sigma_{\mathbf{1}} \cdot \sigma_{\mathbf{2}}\right) \mathbf{k}^{\mathbf{2}}\right]\right\} \\
& +U\left\{\left(\sigma_{\mathbf{1}} \cdot \mathbf{k}^{\prime}\right) \delta\left(\mathbf{r}_{\mathbf{1}}-\mathbf{r}_{\mathbf{2}}\right)\left(\sigma_{\mathbf{2}} \cdot \mathbf{k}\right)-\frac{\mathbf{1}}{\mathbf{3}}\left(\sigma_{\mathbf{1}} \cdot \sigma_{\mathbf{2}}\right) \delta\left(\mathbf{r}_{\mathbf{1}}-\mathbf{r}_{\mathbf{2}}\right)\left[\mathbf{k}^{\prime} \cdot \mathbf{k}\right]\right\},
\end{aligned}
$$


where $k^{\prime}=-\frac{\overleftarrow{\nabla}_{\mathbf{1}}-\overleftarrow{\nabla}_{\mathbf{2}}}{2 i}$ acts on the left while $k=\frac{\vec{\nabla}_{\mathbf{1}}-\vec{\nabla}_{\mathbf{2}}}{2 i}$ acts on the right. Using the formula

$$
(\mathbf{a} \cdot \mathbf{b})(\mathbf{c} \cdot \mathbf{d})=\frac{(\mathbf{a} \cdot \mathbf{c})(\mathbf{b} \cdot \mathbf{d})}{3}+\frac{(\mathbf{a} \times \mathbf{c})(\mathbf{b} \times \mathbf{d})}{2}+\sum_{\mathbf{q}=-2}^{\mathbf{2}}(-\mathbf{1})^{\mathbf{2}-\mathbf{q}}\left[\mathbf{a}^{(\mathbf{1})} \otimes \mathbf{c}^{(\mathbf{1})}\right]_{\mathbf{q}}^{(\mathbf{2})}\left[\mathbf{b}^{(\mathbf{1})} \otimes \mathbf{d}^{(\mathbf{1})}\right]_{-\mathbf{q}}^{(\mathbf{2})}
$$

we can re-write (A1) in such a way that the coupling of both the spatial and spin operators to rank 2 is evident,

$$
\begin{aligned}
V_{\text {tensor }}= & \frac{T}{2}\left\{\sum_{q=-2}^{2}(-1)^{2-q}\left[\sigma_{\mathbf{1}} \otimes \sigma_{\mathbf{2}}\right]_{\mathbf{q}}^{(\mathbf{2})}\left[\mathbf{k}^{\prime} \otimes \mathbf{k}^{\prime}\right]_{-\mathbf{q}}^{(\mathbf{2})} \delta\left(\mathbf{r}_{\mathbf{1}}-\mathbf{r}_{\mathbf{2}}\right)\right. \\
& +\delta\left(\mathbf{r}_{\mathbf{1}}-\mathbf{r}_{\mathbf{2}}\right) \sum_{\mathbf{q}=-\mathbf{2}}^{\mathbf{2}}(-\mathbf{1})^{\mathbf{2}-\mathbf{q}}\left[\left[\sigma_{\mathbf{1}} \otimes \sigma_{\mathbf{2}}\right]_{\mathbf{q}}^{(\mathbf{2})}[\mathbf{k} \otimes \mathbf{k}]_{-\mathbf{q}}^{(\mathbf{2})}\right\} \\
& +U\left\{\sum_{q=-2}^{2}(-1)^{2-q}\left[\sigma_{\mathbf{1}} \otimes \sigma_{\mathbf{2}}\right]_{\mathbf{q}}^{(\mathbf{2})}\left[\mathbf{k}^{\prime} \otimes \mathbf{k}\right]_{-\mathbf{q}}^{(\mathbf{2})} \delta\left(\mathbf{r}_{\mathbf{1}}-\mathbf{r}_{\mathbf{2}}\right)\right\} .
\end{aligned}
$$

For RPA, we wish to calculate the coupled $\mathrm{p}$-h matrix elements defined as

$$
V_{a b c d}^{(J)}=\frac{1}{2 J+1} \sum_{m_{a}, m_{b}, m_{c}, m_{d}, M}(-1)^{j_{c}-m_{c}+j_{b}-m_{b}}\left\langle j_{a} m_{a}, j_{c}-m_{c} \mid J M\right\rangle\left\langle j_{d} m_{d}, j_{b}-m_{b} \mid J M\right\rangle\left\langle a m_{a} b m_{b}|V| c m_{c} d m_{d}\right\rangle .
$$

We use the well-known Pandya relation to re-express the $\mathrm{p}$-h matrix elements in terms of the particle-particle (pp) ones,

$$
V_{a b c d}^{(J)}=\sum_{J^{\prime}}{\widehat{J^{\prime}}}^{2}(-1)^{j_{c}+j_{d}+J^{\prime}}\left\{\begin{array}{lll}
J^{\prime} & j_{b} & j_{a} \\
J & j_{c} & j_{d}
\end{array}\right\}\left\langle\left(j_{a} j_{b}\right) J^{\prime}|V|\left(j_{c} j_{d}\right) J^{\prime}\right\rangle
$$

and change the jj coupling to the LS one, to obtain

$$
\begin{aligned}
V_{a b c d}^{(J)} & =\sum_{J^{\prime}}{\widehat{J^{\prime}}}^{2}(-1)^{j_{c}+j_{d}+J^{\prime}}\left\{\begin{array}{ccc}
J^{\prime} & j_{b} & j_{a} \\
J & j_{c} & j_{d}
\end{array}\right\} \\
& \times \sum_{L, L^{\prime}, S, S^{\prime}} \widehat{j_{a}} \widehat{j_{b}} \widehat{j_{c}} \widehat{j_{d}} \widehat{S} \widehat{S^{\prime}} \widehat{L} \widehat{L^{\prime}}\left\{\begin{array}{ccc}
l_{a} & L & l_{b} \\
j_{a} & J^{\prime} & j_{b} \\
\frac{1}{2} & S & \frac{1}{2}
\end{array}\right\}\left\{\begin{array}{ccc}
l_{c} & L^{\prime} & l_{d} \\
j_{c} & J^{\prime} & j_{d} \\
\frac{1}{2} & S^{\prime} & \frac{1}{2}
\end{array}\right\}\left\langle(L S) J^{\prime}|V|\left(L^{\prime} S^{\prime}\right) J^{\prime}\right\rangle .
\end{aligned}
$$

Then,

$$
\left\langle(L S) J^{\prime} M^{\prime}|V|\left(L^{\prime} S^{\prime}\right) J^{\prime} M^{\prime}\right\rangle=(-1)^{S+J^{\prime}+L^{\prime}}\left\{\begin{array}{lll}
L & S & J^{\prime} \\
S^{\prime} & L^{\prime} & 2
\end{array}\right\}\left\langle S\left\|\left[\sigma_{1} \otimes \sigma_{2}\right]^{(2)}\right\| S^{\prime}\right\rangle\left\langle L\left\|\left[\hat{O}_{1} \otimes \hat{O}_{2}\right]^{(2)}\right\| L^{\prime}\right\rangle
$$

where the operators $\hat{O}_{i}$ acts on the space part of the wavefunction. For the spin part, the reduced matrix element is simply

$$
\left\langle S\left\|\left[\sigma_{1} \otimes \sigma_{2}\right]^{(2)}\right\| S^{\prime}\right\rangle=\sqrt{3 \times 3 \times 5} \times \frac{1}{9} \times \sqrt{6} \times \sqrt{6}=2 \sqrt{5}
$$

while the space part is quite lengthy to evaluate. After applications of the gradient formula and proper recouplings, one arrives at the final result, that is,

$$
\begin{aligned}
\left\langle(L S) J^{\prime}|V|\left(L^{\prime} S^{\prime}\right) J^{\prime}\right\rangle & =\sum_{\alpha, \lambda, \lambda^{\prime}}(-1)^{S+J^{\prime}+L^{\prime}}\left\{\begin{array}{lll}
L & 1 & J^{\prime} \\
1 & L^{\prime} & 2
\end{array}\right\} \times 2 \sqrt{5} \\
& \times\left\{\begin{array}{lll}
l_{a} & l_{b} & L \\
l_{c} & l_{d} & L^{\prime} \\
\lambda^{\prime} & \alpha & 2
\end{array}\right\}{\widehat{\lambda^{\prime}}}^{2} \widehat{\lambda}^{2} \times \widehat{L} \widehat{L^{\prime}} \times 5 \times \frac{1}{4} \sum_{n=1}^{10} v^{(n)},
\end{aligned}
$$


where

$$
\begin{aligned}
v^{(1)} & =\frac{T}{2} \sum_{k, i= \pm 1}(-1)^{\frac{i}{2}+\frac{k}{2}+k+\lambda+\lambda^{\prime}}\left(l_{a}+\frac{i}{2}+\frac{1}{2}\right)^{\frac{1}{2}}\left(l_{a}+i+\frac{k}{2}+\frac{1}{2}\right)^{\frac{1}{2}}\left\{\begin{array}{ccc}
1 & 1 & 2 \\
\lambda^{\prime} & \alpha & \lambda
\end{array}\right\}\left\{\begin{array}{ccc}
l_{c} & l_{a}+i & \lambda \\
1 & \alpha & l_{a}+i+k
\end{array}\right\} \\
& \times\left\{\begin{array}{ccc}
1 & \lambda & \lambda^{\prime} \\
l_{c} & l_{a} & l_{a}+i
\end{array}\right\}\left\langle l_{c}\left\|Y_{\alpha}\right\| l_{a}+i+k\right\rangle\left\langle l_{b}\left\|Y_{\alpha}\right\| l_{d}\right\rangle \int d r r^{2} \phi_{b} \phi_{c}(r) \phi_{d}(r) D_{l_{a}}^{i} D_{l_{a}+i}^{k} \phi_{a}(r),
\end{aligned}
$$

$v^{(2)}$ has the same form as $v^{(1)}$ with the changes $a \longleftrightarrow b$ and $c \longleftrightarrow d, v^{(3)}$ has the same form as $v^{(1)}$ with the change $a \longleftrightarrow c$ and an additional phase $(-1)^{\lambda^{\prime}}$, and $v^{(4)}$ has the same form as $v^{(1)}$ with the changes $a \longleftrightarrow d$ and $c \longleftrightarrow b$ plus the additional phase $(-1)^{\lambda^{\prime}-\alpha}$. Then

$$
\begin{aligned}
& v^{(5)}=U \sum_{k, i= \pm 1}(-1)^{\frac{i}{2}+\frac{k}{2}-i+1+\alpha}\left(l_{a}+\frac{i}{2}+\frac{1}{2}\right)^{\frac{1}{2}}\left(l_{c}+\frac{k}{2}+\frac{1}{2}\right)^{\frac{1}{2}}\left\{\begin{array}{lll}
1 & 1 & 2 \\
\lambda^{\prime} & \alpha & \lambda
\end{array}\right\}\left\{\begin{array}{llll}
l_{c}+k & l_{a} & \lambda \\
1 & & \alpha & l_{a}+i
\end{array}\right\} \\
& \times\left\{\begin{array}{lll}
\lambda & 1 & \lambda^{\prime} \\
l_{c} & l_{a} & l_{c}+k
\end{array}\right\}\left\langle l_{a}+i\left\|Y_{\alpha}\right\| l_{c}+k\right\rangle\left\langle l_{b}\left\|Y_{\alpha}\right\| l_{d}\right\rangle \int d r r^{2} \phi_{b}(r) \phi_{d}(r) D_{l_{a}}^{i} \phi_{a}(r) D_{l_{c}}^{k} \phi_{c}(r),
\end{aligned}
$$

and $v^{(6)}$ has the same form as $v^{(5)}$ with the changes $a \longleftrightarrow b$ and $c \longleftrightarrow d$. Finally,

$$
\begin{aligned}
v^{(7)} & =T \sum_{k, i= \pm 1}(-1)^{\frac{i}{2}+\frac{k}{2}+\lambda^{\prime}}\left(l_{c}+\frac{i}{2}+\frac{1}{2}\right)^{\frac{1}{2}}\left(l_{d}+\frac{k}{2}+\frac{1}{2}\right)^{\frac{1}{2}}\left\{\begin{array}{ccc}
1 & \lambda & \alpha \\
\lambda^{\prime} & 1 & 2
\end{array}\right\}\left\{\begin{array}{ccc}
\lambda & l_{a} & l_{c} \\
l_{c}+i & 1 & \alpha
\end{array}\right\} \\
& \times\left\{\begin{array}{ccc}
\lambda^{\prime} & l_{b} & l_{d} \\
l_{d}+k & 1 & \alpha
\end{array}\right\}\left\langle l_{a}\left\|Y_{\alpha}\right\| l_{c}+i\right\rangle\left\langle l_{b}\left\|Y_{\alpha}\right\| l_{d}+k\right\rangle \int d r r^{2} \phi_{a}(r) \phi_{b}(r) D_{l_{c}}^{i} \phi_{c}(r) D_{l_{d}}^{k} \phi_{d}(r),
\end{aligned}
$$

and $v^{(8)}$ has the same form as $v^{(7)}$ with the changes $c \longleftrightarrow a, d \longleftrightarrow b$ plus the additional phase $(-1)^{\lambda-\lambda^{\prime}}, v^{(9)}$ has the same form as $v^{(7)}$ with the changes $T \longrightarrow U, c \longleftrightarrow a$ plus the additional phase $(-1)^{1+\lambda}$, and $v^{(10)}$ has the same form as $v^{(7)}$ with the changes $T \longrightarrow U, b \longleftrightarrow d$ plus the additional phase $(-1)^{1-\lambda^{\prime}}$.

In all the formulas (A10), (A11) and (A12) the following differential operator is used:

$$
D_{l}^{m}=\frac{d}{d r}+\frac{(-)^{\frac{m}{2}+\frac{l}{2}}\left(l-\frac{m}{2}+\frac{1}{2}\right)}{r} .
$$

\section{APPENDIX B: A SEPARABLE APPROXIMATION FOR THE TENSOR INTERACTION}

The tensor-even term in Eq. (A3) can be written as

$$
V_{\text {tensor }}^{\text {even }}=\frac{T}{2}\left\{\left[\sqrt{5}\left[\sigma_{1} \otimes \sigma_{2}\right]^{(2)} \otimes\left[\mathbf{k}^{\prime} \otimes \mathbf{k}^{\prime}\right]^{(2)}\right]^{(0)}+\sqrt{5}\left[\left[\sigma_{1} \otimes \sigma_{2}\right]^{(2)} \otimes[\mathbf{k} \otimes \mathbf{k}]^{(2)}\right]^{(0)}\right\} \delta\left(\mathbf{r}_{\mathbf{1}}-\mathbf{r}_{2}\right) .
$$

We assume that the contributions including gradients acting twice on the same wavefunction $\left(\sim \nabla_{1} \cdot \nabla_{1}\right.$ or $\left.\sim \nabla_{2} \cdot \nabla_{2}\right)$ are less important than the terms $\sim \nabla_{1} \cdot \nabla_{2}$ or $\sim \nabla_{2} \cdot \nabla_{1}$, and we neglect them. With this approximation, and the multipole expansion of the $\delta$-function, we can write one of the leading terms as

$$
V_{\text {tensor }}^{\text {even }}(\text { term } 1)=\frac{T}{2} \sum_{\ell} \sqrt{5} \hat{\ell}(-)^{\ell} \frac{1}{4}\left\{\left[\sigma_{1} \otimes \sigma_{2}\right]^{(2)} \otimes\left[\nabla_{1} \otimes \nabla_{\mathbf{2}}\right]^{(2)}\right\}^{(0)}\left[Y_{\ell}(1) \otimes Y_{\ell}(2)\right]^{(0)} .
$$

We apply standard angular momentum techniques to express this term of the interaction as a sum of separable terms, that is, terms made up with a product of two operators acting respectively only on particle 1 and particle 2 . The result is

$$
V_{\text {tensor }}^{\text {even }}(\text { term } 1)=\frac{5 T}{8} \sum_{\ell, \lambda, \lambda^{\prime}, k}(-)^{\lambda+\lambda^{\prime}+k} \hat{\lambda} \hat{\lambda}^{\prime} \hat{k}\left\{\begin{array}{ccc}
\lambda & \lambda^{\prime} & 2 \\
1 & 1 & \ell
\end{array}\right\}\left\{\begin{array}{ccc}
1 & 1 & 2 \\
\lambda^{\prime} & \lambda & k
\end{array}\right\}\left[\hat{O}_{\lambda^{\prime}, k}(1) \otimes \hat{O}_{\lambda, k}(2)\right]^{(0)},
$$

where the operator is

$$
\hat{O}_{\lambda, k}(i)=\left[\sigma_{i} \otimes\left(\nabla_{i} \otimes Y_{\ell}(i)\right)^{(\lambda)}\right]^{(k)} .
$$


If we calculate the p-h matrix elements coupled to $J$ [cf. the previous appendix, Eq. (A44)], then $k=J$ and

$$
V_{p^{\prime} h h^{\prime} p}^{(J)}(\operatorname{term} 1)=\frac{5 T}{8} \sum_{\ell, \lambda, \lambda^{\prime}}(-)^{\lambda+\lambda^{\prime}+k} \hat{\lambda} \hat{\lambda}^{\prime} \hat{k}\left\{\begin{array}{ccc}
\lambda & \lambda^{\prime} & 2 \\
1 & 1 & \ell
\end{array}\right\}\left\{\begin{array}{ccc}
1 & 1 & 2 \\
\lambda^{\prime} & \lambda & k
\end{array}\right\}\left\langle p^{\prime}\left|\hat{O}_{\lambda^{\prime}, k}\right| h^{\prime}\right\rangle\left\langle h\left|\hat{O}_{\lambda, k}\right| p\right\rangle
$$

We then analyze the quantity

$$
\left\langle p^{\prime}\left|\hat{O}_{\lambda^{\prime}, k}\right| h^{\prime}\right\rangle\left\langle h\left|\hat{O}_{\lambda, k}\right| p\right\rangle .
$$

Due to the time-reversal properties of the operator (Bי $)$, this is equal to

$$
-\left\langle p^{\prime}\left|\hat{O}_{\lambda^{\prime}, k}\right| h^{\prime}\right\rangle\left\langle p\left|\hat{O}_{\lambda, k}\right| h\right\rangle
$$

(cf., e.g., p. 312-313 of [25]). To make an estimate of the sign of the diagonal matrix elements, we obtain from (B5) and (B7)

$$
V_{p h h p}^{(J)}\left(\text { term 1) }=-\frac{5 T}{8} \sum_{\ell, \lambda, \lambda^{\prime}}(-)^{\lambda+\lambda^{\prime}+k} \hat{\lambda} \hat{\lambda}^{\prime} \hat{k}\left\{\begin{array}{ccc}
\lambda & \lambda^{\prime} & 2 \\
1 & 1 & \ell
\end{array}\right\}\left\{\begin{array}{ccc}
1 & 1 & 2 \\
\lambda^{\prime} & \lambda & k
\end{array}\right\}\left\langle p\left|\hat{O}_{\lambda^{\prime}, k}\right| h\right\rangle\left\langle p\left|\hat{O}_{\lambda, k}\right| h\right\rangle .\right.
$$

We apply this latter equation for two typical cases (of interest for the low-lying spectroscopy of nuclei), namely the natural parity case $J^{\pi}=2^{+}$and the unnatural parity case $J^{\pi}=1^{+}$. We obtain

$$
\begin{aligned}
& \operatorname{sign}\left(V_{p h h}^{\left(2^{+}\right)}(\text {even, term } 1)\right)=-\operatorname{sign}(T), \\
& \operatorname{sign}\left(V_{p h h p}^{\left(1^{+}\right)}(\text {even, term } 1)\right)=+\operatorname{sign}(T) .
\end{aligned}
$$

Exactly the same kind of study can be performed for the tensor-odd part of the interaction, namely for the term

$$
V_{\text {tensor }}^{\text {odd }}=U\left\{\sqrt{5}\left[\left[\sigma_{1} \otimes \sigma_{2}\right]^{(2)} \otimes\left[\mathbf{k}^{\prime} \otimes \mathbf{k}\right]^{(2)}\right]^{(0)}\right\} \delta\left(\mathbf{r}_{1}-\mathbf{r}_{2}\right) .
$$

In this case, we assume that the leading term is $\sim \nabla_{1}^{\prime} \cdot \nabla_{2}$ (where the prime means that the gradient is acting at left). The equation corresponding to (B2) is

$$
V_{\text {tensor }}^{\text {odd }}(\text { term } 1)=-\frac{U}{4} \sum_{\ell} \sqrt{5} \hat{\ell}(-)^{\ell}\left\{\left[\sigma_{1} \otimes \sigma_{2}\right]^{(2)} \otimes\left[\nabla_{1}^{\prime} \otimes \nabla_{\mathbf{2}}\right]^{(2)}\right\}^{(0)}\left[Y_{\ell}(1) \otimes Y_{\ell}(2)\right]^{(0)} .
$$

By developing the same procedure outlined for the even term, we come to the conclusion that

$$
\begin{aligned}
& \operatorname{sign}\left(V_{p h h p}^{\left(2^{+}\right)}(\text {odd }, \text { term } 1)\right)=+\operatorname{sign}(U), \\
& \operatorname{sign}\left(V_{p h h p}^{\left(1^{+}\right)}(\text {odd, term } 1)\right)=+\operatorname{sign}(U) .
\end{aligned}
$$

We can use Eqs. (B9), (B10), (B13) and (B14) as a useful guideline. In fact, these equations correspond to direct (i.e., non antisymmetrized) matrix elements. The antisymmetrized interaction can be written as

$$
V_{\text {tensor }}\left(1-P_{M} P_{\sigma} P_{\tau}\right),
$$

where the position, spin and isospin exchange operators $P_{M}, P_{\sigma}$ and $P_{\tau}$ have been introduced. Since the tensor interaction acts only among spin-triplet states, $P_{\sigma}=1$. By definition, $P_{M}=+1(-1)$ in the case of the even (odd) tensor term. Therefore, if we call for simplicity

$$
\begin{aligned}
V(\text { even }) & =V_{p h h p}^{\left(J^{\pi}\right)}(\text { even }), \\
V(\text { odd }) & =V_{p h h p}^{\left(J^{\pi}\right)}(\text { odd }),
\end{aligned}
$$

it is simple to arrive at

$$
\begin{aligned}
V_{p h h p}^{\left(J^{\pi}\right)} & =\left(\frac{1}{2} V(\text { even })+\frac{3}{2} V(\text { odd })\right) & \text { IS part } \\
& +\left(-\frac{1}{2} V(\text { even })+\frac{1}{2} V(\text { odd })\right)\left\langle\tau_{1} \cdot \tau_{2}\right\rangle & \text { IV part. }
\end{aligned}
$$


The sign of the residual interaction can be understood by combining this latter equation with the previous ones. This is at least true when the different terms add up coherently; in case of cancellations, of course, the qualitative arguments of this Appendix may not be valid.

[1] E. Chabanat, P. Bonche, P. Haensel, J. Meyer, R. Schaeffer, Nucl. Phys. A635, 231 (1998).

[2] M. Bender, P.-H. Heenen, P.-G. Reinhard, Rev. Mod. Phys. 75, 121 (2003).

[3] G. Colò, in Bulk Nuclear Properties. Proceedings of the Fifth ANL/MSU/JINA/INT FRIB Workshop, edited by P. Danielewicz (American Institute of Physics, Melville, New York, 2009), and arXiv:0902.3739.

[4] B. A. Brown, T. Duguet, T. Otsuka, D. Abe, and T. Suzuki, Phys. Rev. C 74, 061303(R) (2006).

[5] J. Dobaczewski, in Proceedings of the Third ANL/MSU/JINA/INT RIA Workshop, edited by T. Duguet, H. Esbensen, K. M. Nollett, and C. D. Roberts (World Scientific, Singapore, 2006).

[6] G. Colò, H. Sagawa, S. Fracasso and P.F. Bortignon, Phys. Lett. B646, 227 (2007) [see also: Phys. Lett. B668, 457 (2008)].

[7] D. M. Brink and F. Stancu, Phys. Rev. C 75, 064311 (2007).

[8] T. Lesinski, M. Bender, K. Bennaceur, T. Duguet, and J. Meyer, Phys. Rev. C 76, 014312 (2007).

[9] M. Grasso, Z. Ma, E. Khan, J. Margueron, and N. Van Giai, Phys. Rev. C 76, 044319 (2007).

[10] W. Zou, G. Colò, Z. Ma, H. Sagawa and P.F. Bortignon, Phys. Rev. C77, 014314 (2008).

[11] M. Zalewski, J. Dobaczewski, W. Satula, and T. R. Werner, Phys. Rev. C 77, 024316 (2008).

[12] T. Otsuka, T. Suzuki, R. Fujimoto, H. Grawe, and Y. Akaishi, Phys. Rev. Lett. 95, 232502 (2005); T. Otsuka, T. Matsuo, and D. Abe, Phys. Rev. Lett. 97, 162501 (2006).

[13] G. Colò, P. F. Bortignon, S. Fracasso and N. Van Giai, Nucl. Phys. A788, 137c (2007).

[14] J. Li, G. Colò, J. Meng, Phys. Rev. C 78, 064304 (2008).

[15] V. De Donno, G. Co', C. Maieron, M. Anguiano, A. M. Lallena, M. Moreno Torres, Phys. Rev. C79, 044311 (2009).

[16] D. Davesne, M. Martini, K. Bennaceur, and J. Meyer, arXiv:0906.1927v1.

[17] K.-F. Liu, H.-D. Luo, Z.-Y. Ma, M. Feng, Q.-B. Shen, Nucl. Phys. A534, 48 (1991).

[18] T. Kibédi and R. H. Spear, At. Data Nucl. Data Tables 80, 35 (2002).

[19] S. Raman, C. W. Nestor, et al., At. Data Nucl. Data Tables 78, 1 (2001).

[20] C. L. Bai, H. Sagawa, H. Q. Zhang, X. Z. Zhang, G. Colò, and F. R. Xu, Phys. Lett. B675, 1 (2009); C. L. Bai, H. Q. Zhang, X. Z. Zhang, F. R. Xu, H. Sagawa, and G. Colò, Phys. Rev. C79, 041301(R) (2009).

[21] S. Fracasso and G. Colò, Phys. Rev. C76, 044307 (2007).

[22] S.K. Nanda, C. Glashausser, K.W. Jones, J.A. McGill, T.A. Carey, J.B. McClelland, J.M. Moss, S.J. Seestrom-Morris, J.R. Comfort, S. Levenson, R. Segel, H. Ohnuma, Phys. Rev. C29, 660 (1984).

[23] T. Shizuma, T. Hayakawa, H. Ohgaki, H. Toyokawa, T. Komatsubara, N. Kikuzawa, A. Tamii, and H. Nakada, Phys. Rev. C78, 061303(R) (2008).

[24] T. H. R. Skyrme, Philos. Mag. 1, 1043 (1956); J. S. Bell and T. H. R. Skyrme, Philos. Mag. 1, 10455 (1956); T. H. R. Skyrme, Nucl. Phys. 9, 615 (1958); 9, 635 (1958).

[25] A. Bohr and B. R. Mottelson, Nuclear Structure, vol. I (W.A. Benjamin, Reading, 1969). 
TABLE I: Parameters of the effective interactions used in the HF plus RPA calculations. The set SLy 5 has been introduced in [1], and it is here supplemented by the tensor force parameters $T$ and $U$ which have been introduced in [6]. The set T44 is taken from [8]. The notation is the same as in [1] for the Skyrme parameters, while $T$ and $U$ are defined as in [6].

\begin{tabular}{ccc}
\hline \hline & SLy5+(T,U) & T44 \\
\hline$t_{0}\left[\mathrm{MeV} \mathrm{fm}^{3}\right]$ & -2484.88 & -2485.670 \\
$t_{1}\left[\mathrm{MeV} \mathrm{fm}^{5}\right]$ & 483.13 & 494.477 \\
$t_{2}\left[\mathrm{MeV} \mathrm{fm}^{5}\right]$ & -549.40 & -337.961 \\
$t_{3}\left[\mathrm{MeV} \mathrm{fm}^{3+3 \sigma}\right]$ & 13763.0 & 13794.7 \\
$x_{0}$ & 0.778 & 0.721557 \\
$x_{1}$ & -0.328 & -0.661848 \\
$x_{2}$ & -1.000 & -0.803184 \\
$x_{3}$ & 1.267 & 1.175908 \\
$\sigma$ & $1 / 6$ & $1 / 6$ \\
$W_{0}\left[\mathrm{MeV} \mathrm{fm}^{5}\right]$ & 126.0 & 161.367 \\
\hline$T\left[\mathrm{MeV} \mathrm{fm}^{5}\right]$ & 888.0 & 520.983 \\
$U\left[\mathrm{MeV} \mathrm{fm}^{5}\right]$ & -408.0 & 21.522 \\
\hline \hline
\end{tabular}

TABLE II: Properties of the low-lying quadrupole states in ${ }^{48} \mathrm{Ca}$ and ${ }^{208} \mathrm{~Pb}$. Energies and $\mathrm{B}(\mathrm{E} 2)$ values from the RPA calculations are compared with experimental data from Ref. [19].

\begin{tabular}{ccc}
\hline \hline & Energy $[\mathrm{MeV}]$ & $\mathrm{B}(\mathrm{E} 2)\left[\mathrm{e}^{2} \mathrm{fm}{ }^{4}\right]$ \\
\hline${ }^{48} \mathrm{Ca}$ & & 56.5 \\
\hline SLy5 (no tensor) & 3.05 & 52.9 \\
SLy5 (with tensor) & 3.88 & 53.2 \\
T44 (no tensor) & 3.35 & 51.8 \\
T44 (with tensor) & 3.17 & 95.0 \\
Exp. & 3.83 & $2.9410^{3}$ \\
\hline${ }^{208}$ Pb & & $3.0710^{3}$ \\
\hline SLy5 (no tensor) & 4.89 & $2.8610^{3}$ \\
SLy5 (with tensor) & 5.06 & $2.7010^{3}$ \\
T44 (no tensor) & 5.04 & $2.9710^{3}$ \\
T44 (with tensor) & 4.49 & 4.07 \\
Exp. & & \\
\hline \hline
\end{tabular}


TABLE III: The same as Table $\amalg$ for the case of the low-lying octupole states. In this case the nuclei are ${ }^{40} \mathrm{Ca},{ }^{48} \mathrm{Ca}$ and ${ }^{208} \mathrm{~Pb}$. The experimental data are from Ref. [18].

\begin{tabular}{|c|c|c|}
\hline & Energy $[\mathrm{MeV}]$ & $\mathrm{B}(\mathrm{E} 3)\left[\mathrm{e}^{2} \mathrm{fm}^{6}\right]$ \\
\hline \multicolumn{3}{|l|}{${ }^{40} \mathrm{Ca}$} \\
\hline SLy5 (no tensor) & 3.78 & $1.3210^{4}$ \\
\hline T44 (no tensor) & 1.36 & $1.2310^{4}$ \\
\hline T44 (with tensor) & 1.22 & $1.0310^{4}$ \\
\hline SLy5 (no tensor) & 4.78 & $0.5410^{4}$ \\
\hline SLy5 (with tensor) & 6.16 & $0.6610^{4}$ \\
\hline T44 (no tensor) & 3.44 & $0.4210^{4}$ \\
\hline T44 (with tensor) & 4.93 & $0.3410^{4}$ \\
\hline Exp. & 4.51 & $1.0010^{4}$ \\
\hline T44 (no tensor) & 3.49 & $5.8710^{5}$ \\
\hline T44 (with tensor) & 3.19 & $6.2910^{5}$ \\
\hline Exp. & 2.61 & $(5.30 \pm 0.30) 10^{5}$ \\
\hline
\end{tabular}



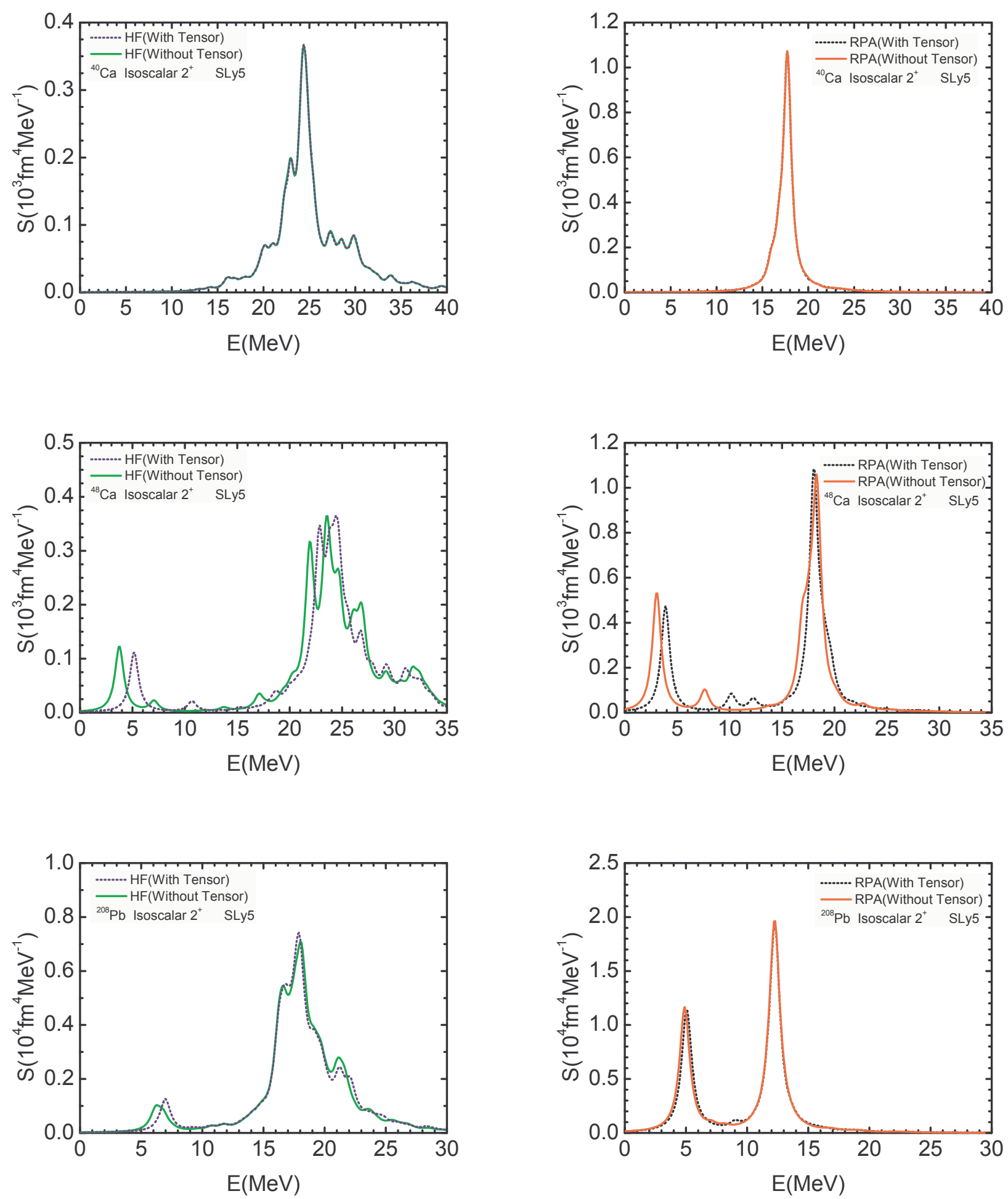

FIG. 1: Unperturbed (left panels) and RPA (right panels) strength functions associated with the isoscalar quadrupole operator, in the case of ${ }^{40} \mathrm{Ca},{ }^{48} \mathrm{Ca}$ and ${ }^{208} \mathrm{~Pb}$. We display results both with and without the inclusion of the tensor force in the case of the force SLy5 plus the $T$ and $U$ parameters of [6] (first column of Table II). The discrete RPA peaks have been smeared out by using Lorentzian functions which have $1 \mathrm{MeV}$ width. 

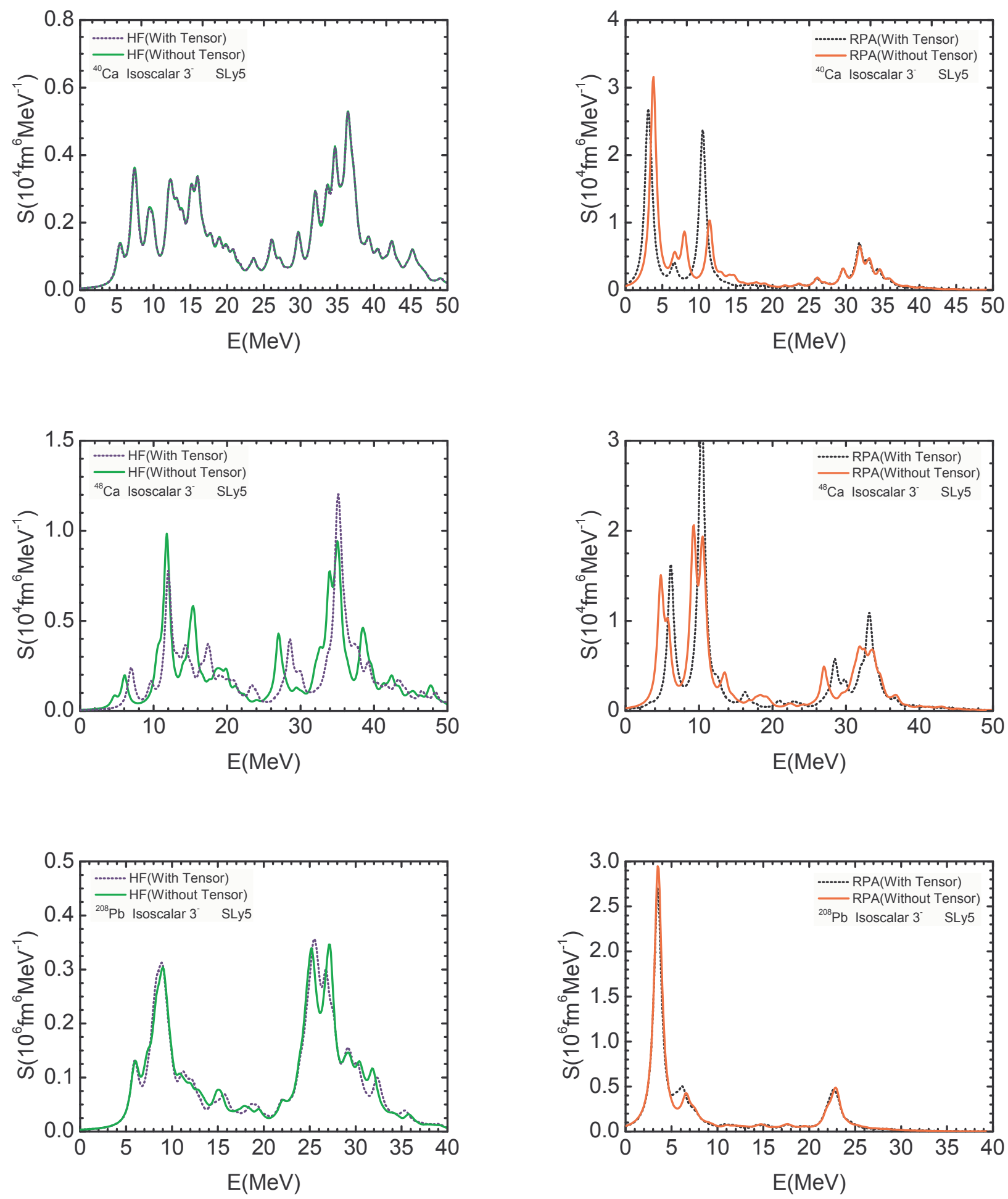

FIG. 2: The same as Fig. 1 in the case of the octupole response. 

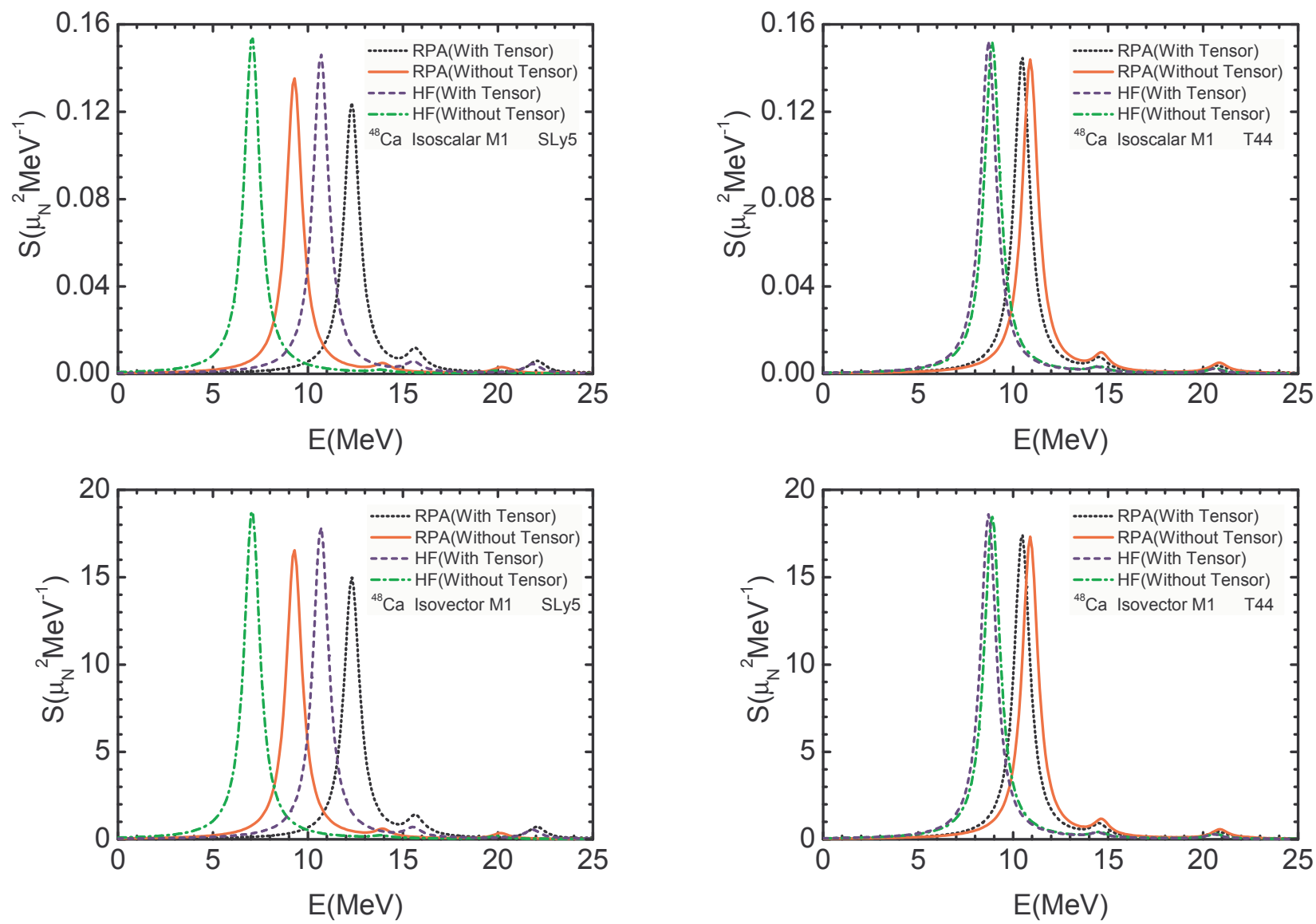

FIG. 3: Unperturbed and RPA strength functions in ${ }^{48} \mathrm{Ca}$ associated with the isoscalar and isovector M1 operators defined by Eqs. (3) and (4). We display results both with and without the inclusion of the tensor force, in the case of SLy5 plus the $T$ and $U$ parameters of [6] (left panels) and in the case of the T44 parameter set (right panels). The discrete RPA peaks have been smeared out by using Lorentzian functions which have $1 \mathrm{MeV}$ width. 

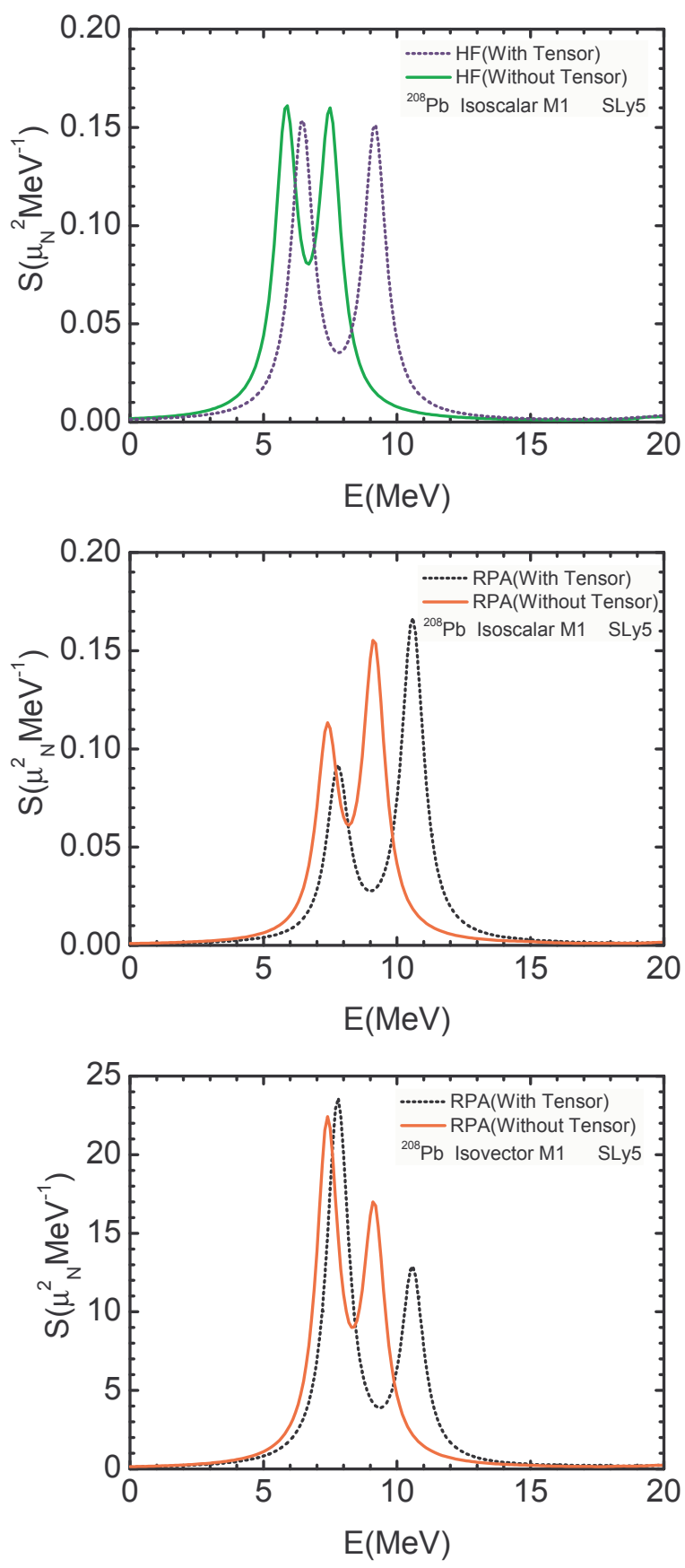

FIG. 4: Unperturbed (upper panel) and RPA (middle and lower panels) strength functions in ${ }^{208} \mathrm{~Pb}$ associated with the isoscalar and isovector M1 operators defined by Eqs. (3) and (44). We display results both with and without the inclusion of the tensor terms, in the case of the force SLy5 plus the $T$ and $U$ parameters of $[\underline{6}$ ] (first column of Table $\llbracket$ ). The discrete RPA peaks have been smeared out by using Lorentzian functions which have $1 \mathrm{MeV}$ width. 

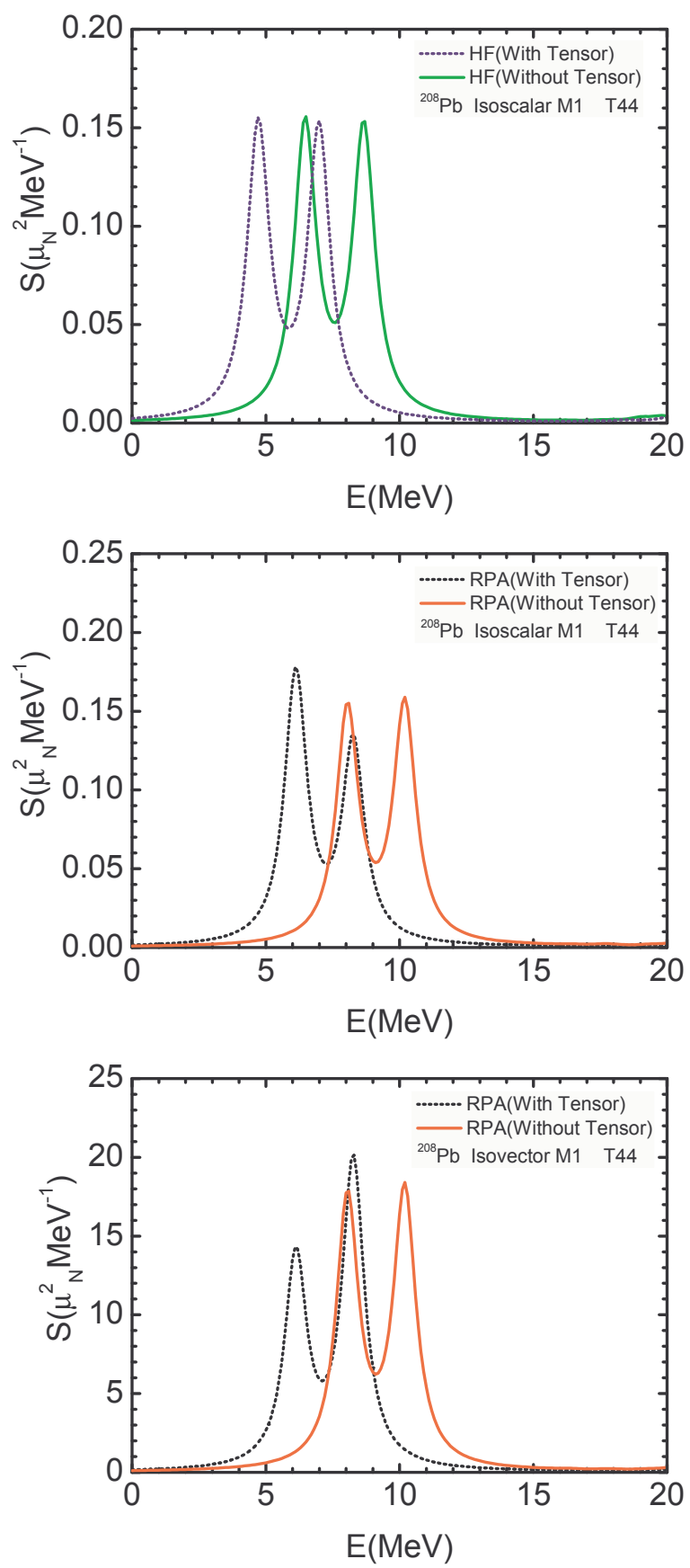

FIG. 5: The same as Fig. 4 in the case of the T44 parameter set (second column of Table \). 